\title{
Whole-mount imaging of the mouse hindlimb vasculature using the lipophilic carbocyanine dye DiI.
}

Jeffrey Boden ${ }^{1,2}$, Jianqin Wei, George McNamara ${ }^{3}$, Hans Layman $^{4}$, Midhat Abdulreda ${ }^{3}$, Fotios Andreopoulos ${ }^{4}$, and Keith A. Webster ${ }^{1,2}$

${ }^{1}$ Department of Molecular and Cellular Pharmacology, ${ }^{2}$ Vascular Biology Institute, ${ }^{3}$ Diabetes Research Institute, and ${ }^{4}$ Department of Bioengineering, University of Miami Miller School of Medicine, Miami, FL, USA

The availability of transgenic disease backgrounds and the accessibility of molecular research reagents have contributed to make the mouse ischemic hindlimb the model of choice for many studies of angiogenesis, and to investigate new treatments for peripheral artery disease. A limitation of these models involves our inability to easily visualize the regenerated vascular architecture. Approaches such as micro-computed tomography and micro-angiography are expensive, technically demanding and not available to many laboratories. Here we describe a rapid and inexpensive adaptation of a vascular staining procedure for precise imaging of the mouse hindlimb vasculature. We introduced two technical modifications and an analytical extension to the original method including (i) pre-skinning of the muscle prior to fixation that preserves tissue integrity, (ii) mild pressure-desiccation subsequent to fixing that enhances resolution and image penetration, and (iii) reconstruction of confocal data into 3D images. The procedure provides resolution that is equivalent or superior to other approaches at a fraction of the cost, time and technology required.

BioTechniques 52:1-4 (July 2012) doi 10.2144/000113907

Keywords: ischemia; DiI; perfusion; vascular; imaging; hindlimb

The mouse hindlimb is the most widely used model to assess vascular responses to ischemia and test new strategies for the treatment of peripheral artery disease (1-4). In these models, the femoral artery (FA) is excised, test therapies are administered, and recovery is followed over a period of weeks (1). A drawback to the mouse models is a limitation in available technology to image and characterize the vasculature directly. Typically, laser Doppler imaging and capillary density quantification by immunostaining of tissue sections are used to assess recovery. These measurements do not provide information on vasculature organization or remodeling of vessels. Vascular architecture is routinely monitored by contrast angiography in larger animals and humans (5). For the mouse such measurements require sophisticated instrumentation that for many laboratories is prohibitively expensive $(\$ 0.5 \mathrm{M}-$ $3 \mathrm{M})$, technically demanding, and both time- and labor-intensive. Techniques with the capability to image the mouse vasculature include micro-computed tomography (6), magnetic resonance imaging (7), X-ray angiography (8), particle perfusion (9), Doppler micro-angiography (10), and vascular casting (11). With the possible exception of micro-CT, most of these techniques are also limited in the simultaneous resolution of capillaries and arteries in the mouse.

Here, we provide a simple, inexpensive, and rapid alternative to these techniques that allows precise visualization of the vasculature within the sagittal plane of the mouse hindlimb in whole-mount preparations using modifications of the procedure described by Li et al. (12). The procedure takes advantage of the hydrophobic partitioning of the lipophilic carbocyanine dye $1,1^{\prime}$-dioctadecyl-3,3,3',3'tetramethylindocarbocyanine perchlorate (DiI) that incorporates into endothelial cells and undergoes lateral diffusion (12). Our adaptations include the following:

Step 1: The vascular system was flushed with PBS and stained with DiI as described in the original protocol (12). Then prior to fixation, the limb was skinned to expose the adductor muscles. To do this, forceps were used to lift the skin overlying the ankle. An incision was made across the medial sagittal plane of the limb up to the groin. The skin was cut circumferentially around the proximal portion of the limb and distally where the foot and ankle join. The edges of the major sagittal cut were

\section{Method summary:}

A protocol is described to generate 3D quantifiable images of the entire mouse hindlimb vasculature. (i) The vascular system is flushed sequentially with PBS and stained with DiI. (ii) The limb is skinned. (iii) The vasculature is infused with $4 \%$ PFA. (iv) The limb is severed and sandwiched between micro glass coverslips and compressed. ( $v$ ) Partial desiccation is implemented by blowing filtered dry air between the plates. (vi) The limb surfaces are imaged on a confocal microscope and reconstructed into composite 35-5x fields using Zen software. 3D composites of stacked Z-series are rendered using Volocity software. 
A

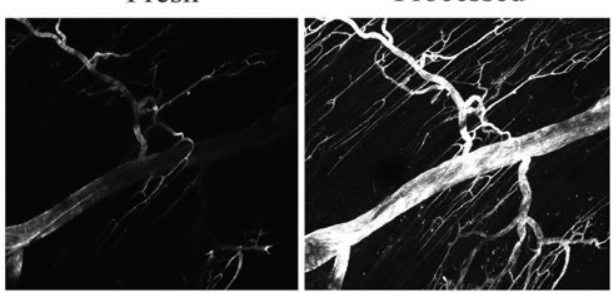

Fresh-enhanced

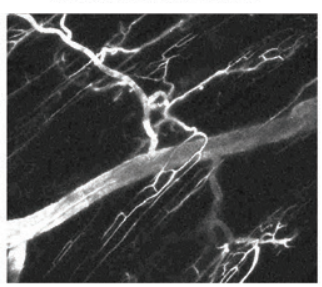

B
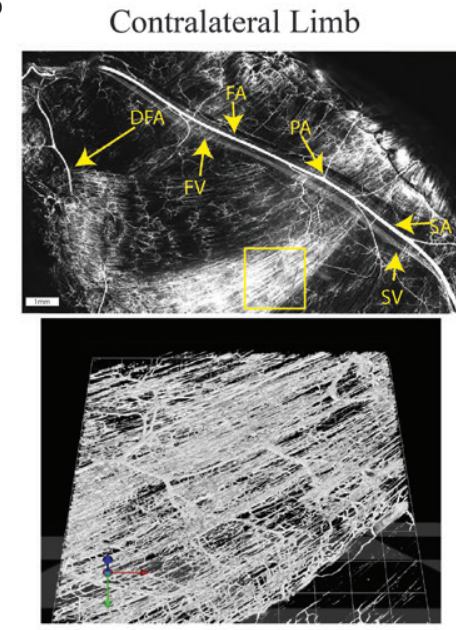

Ischemia 1-Week
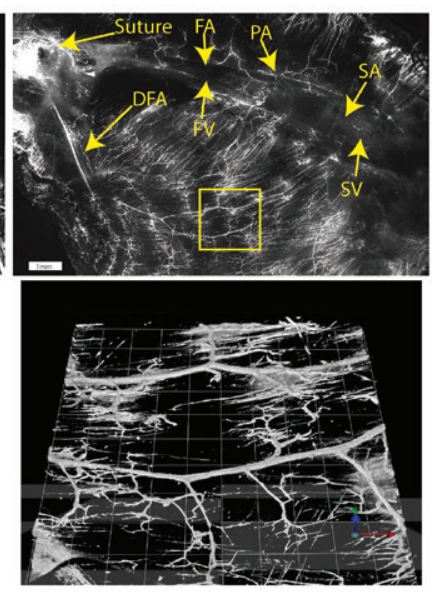

D

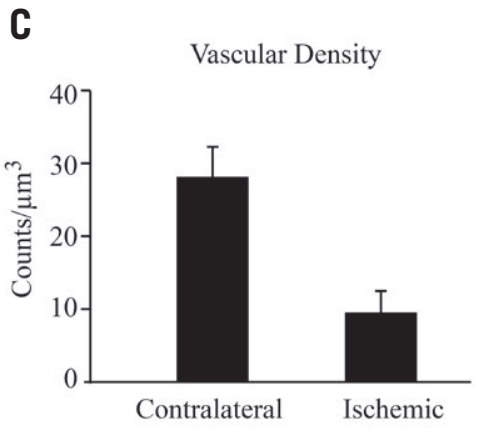

Contralateral
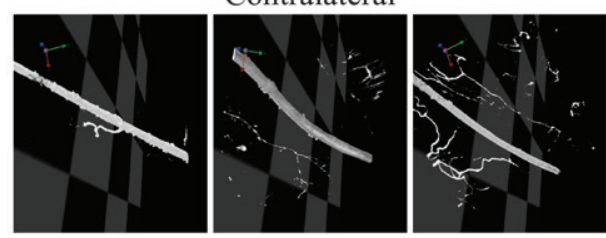

Ischemia
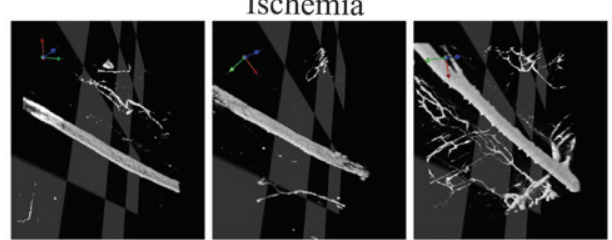

E DFA Diameter

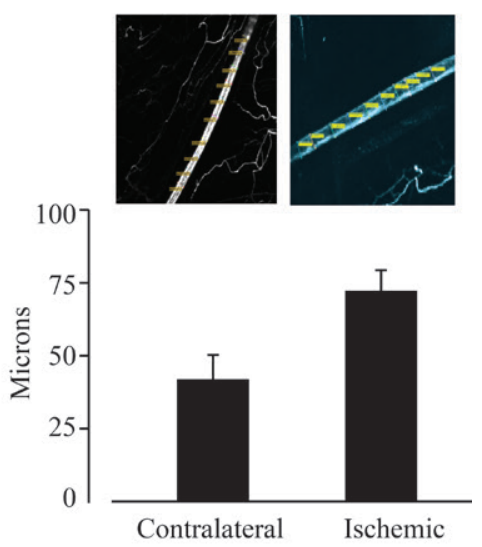

Figure 1. Comparison of vasculature in normal versus ischemic hindlimb of c57/bl6 mice. (A) Section of DFA before and after compression/desiccation processing. Top panels were imaged with identical settings; bottom panel shows Adobe Photoshop-enhanced brightness/contrast of the fresh sample to allow a comparison of the vessel images and evaluate the effects of processing on vessel dimensions. (B) Composite $5 \times$ images of the superficial femoral artery and vein, deep femoral artery, popliteal artery and saphenous artery and vein are shown in normal (left) and ischemic (right) hindlimb 1-week after femoral artery ligation/excision surgery. Composite 10x fields of view (lower panels, which correspond to the yellow boxes in the upper panels) highlight regions of the gastrocnemicus vasculature that were analyzed for vascular density and are rendered in an XYZ plane. DFA, deep femoral artery; FA/FV, superficial femoral artery/vein; PA, popliteal artery; SA/SV, saphenous artery/vein. (C) Volumetric analysis of composite Dil images of 10x fields of view ( $n=$ 16 FOV, $\mathrm{n}=3$ mice) demonstrate 2.7 times higher density of Dil fluorescence per $\mu \mathrm{m}^{3}$ in normal gastrocnemicus muscle. ${ }^{*} P<0.01 ; \pm$ standard deviation. (D) $10 \times$ fields of view of the deep femoral artery in normal and ischemic hindlimbs along the X-Y-Z axes. (E) Cross sectional measurements of the DFA were determined at 10 locations and a mean diameter for each limb was calculated ( $n=3$ mice per group; ${ }^{*} P<0.05, \pm$ standard deviation).

then gently pulled around the back of the limb. After skinning, the hindlimb was perfused with $4 \%$ PFA $(5 \mathrm{~mL})$ by hand using interchangeable $10 \mathrm{~mL}$ syringes and a butterfly needle at a flow rate of 1-2 mL/ min. After fixing, the limb was severed from the trunk, rinsed, and fat and connective tissue dissected. The arteria epigastrica were severed at the point where they meet the FA.

Step 2: The moistened limb was sandwiched between two $43 \times 50 \mathrm{~mm}$ microglass coverslips and compressed between the glass by applying a small $(<200 \mathrm{~g})$ weight uniformly over the coverslip, sufficient to maintain tight contact between the glass and the muscle surface. Compression and drying were performed simultaneously. Air was blown perpendicularly to the plane of the glass at a rate of 28.3 LPM through silicone tubing attached to in-house air for 2-3 h. This process reduces cross-sectional thickness and enhances tissue clarity without requiring optical clearing that can disrupt lipophilic stains (13).

Step 3: Samples were imaged on a Zeiss LSM 710 confocal microscope using optimal step size settings. The entire surface of the upper limb was imaged and reconstructed into a composite of $35-5 x$ fields using Zen software. 3D composites of stacked $\mathrm{Z}$-series were rendered using
Volocity software (Improvision, PerkinElmer, Waltham, MA) (14).

During compression-desiccation the limb loses about $25 \%$ of mass but the vascular architecture remains intact, there are minimal changes in vessel shape, and resolution is dramatically enhanced (Figure 1A). These results demonstrate the improvement in vascular imaging obtained through our modifications over the original protocol described by Li et al. (12).

To illustrate the utility of our technique, we quantified changes in capillary density and arterial diameter in the adductor muscles of C57/BL6 mice at an early time point after ischemia. As shown in Figure 
A

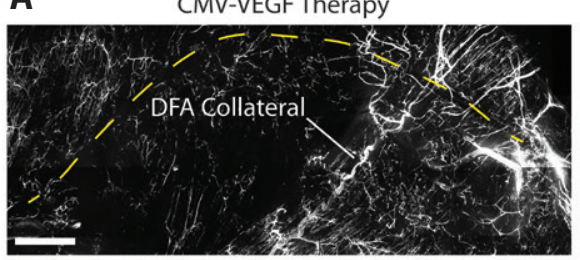

B

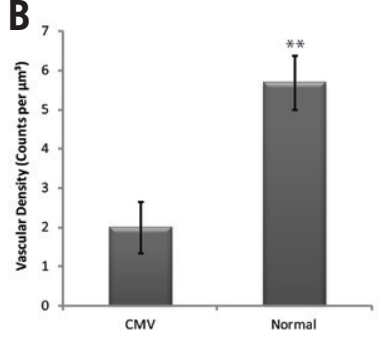

C

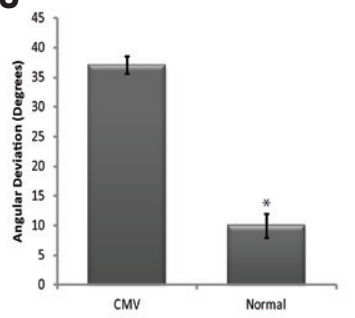

Normal Limb

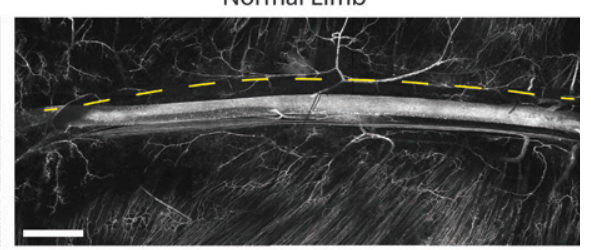

D

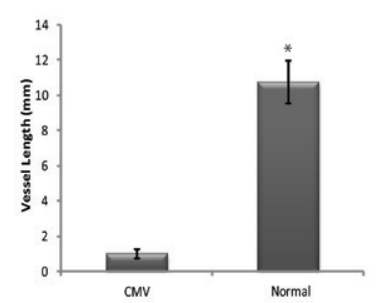

Figure 2. Dil analysis of BALB/c mouse ischemic hindlimb after AAV-VEGF gene therapy. Hindlimbs of $\mathrm{BALB} / \mathrm{c}$ mice were made ischemic by ligation and excision of the FA as described previously (15). AAV-CMV-VEGF or PBS was delivered at 8 injection sites around the excised FA at the time of surgery. (A) Composite Dil images of multiple $10 \times$ fields of view are representative of vessel regeneration along the femoral tract 8 months after FA excision and delivery of an adeno-associated viral (AAV) vector expressing CMV-VEGF (left) compared with a normal (no surgery) limb (right) (scale bars $=1 \mathrm{~mm}$ ). (B-D) Stacked Z-series images were analyzed using Volocity statistical software to quantify total vessel density $(B)$, directionality $(C)$ and length $(D)$ relative to the femoral nerve. The volumetric analysis revealed 2.8 times greater vessel density in normal limb compared with the ischemic limb and CMVVEGF gene therapy (B). To measure vessel length and direction, vessels were traced in the Volocity program and readings of bearing (C) and length (D) were recorded. The path of the femoral nerve was traced in each composite image to serve as a control measure of direction (equal to 0 ) and length (dashed yellow line). By these analyses limbs treated with AAV-CMV-VEGF gene therapy following FA excision develop small vessels that deviate significantly from the path of the femoral nerve suggesting that the regenerated vasculature is disorganized. All values are presented as mean \pm SEM ${ }^{* *} P<$ $0.0001,{ }^{*} P<0.05$.

$1 \mathrm{~B}$, the rich supply of capillaries emanating from the FA close to the popliteal branch is eliminated by FA excision and accounts at least in part for the loss in capillary density by ischemia; 16 separate fields of view from three different mice were used to obtain the values of mean vessel density shown in Figure $1 C$. The results suggest that capillary regeneration at this time point does not compensate for local ischemia-induced loss. Figure 1D (and see online movies in Supplementary materials), shows that ischemia caused significantly increased diameter of the DFA. The average diameter 1-week after femoral artery excision was increased $72 \%$ compared with the contralateral (Figure 1E). This result is in agreement with a previous report on ischemia-induced enlargement of the DFA (9).

As a second illustration of the utility of the method we quantified additional vascular parameters at a late time point after ischemia in the hindlimb of BALB/c mice subjected to concurrent gene therapy. Unlike C57/BL6, the hindlimbs of BALB/c mice are defective in endogenous collateral regeneration (15), and as a consequence the lower limb rapidly auto-amputates below the knee after FA excision. We found that AAV-CMV-VEGF therapy in the
$\mathrm{BALB} / \mathrm{c}$ model enhanced angiogenesis after FA excision but did not support limb salvage despite sustained VEGF expression (results not shown). The long-term effects of sustained VEGF expression by AAV on the mouse hindlimb vasculature are not known. Therefore we used the DiI staining method to analyze these at 8 months following treatment. Figure 2A shows examples of age-matched limbs and in Figures 2B-2D vascular parameters were quantified. We found that the mean vascular density in normal limbs was 2.8 fold greater than in the ischemiaVEGF treatments (Figure 2B; $P<0.0001$ ). Measurements for bearing (vessel direction relative to the femoral nerve) (Figure 2C) and length of large vessels (Figure 2D) were obtained by using the line tool of Volocity. The analyses show that the vasculature created by AAV-CMV-VEGF therapy diverges significantly in terms of vessel quantity, size, and orientation compared with the original vasculature. ,We found that ischemic limbs treated with PBS were similar to the VEGF treatments (data not shown), suggesting absence of any sustained effects of AAV-CMV-VEGF on the vasculature. These results are consistent with previous findings that VEGF gene therapy does not support the production of new collateral vessels (16) and further indicate that chronic VEGF overexpression by AAV in this model does not enhance capillary density.

Supplementary Figure S1 shows a typical composite DiI image of an intact femoral artery from a C57/BL6 mouse with enlarged sections identified by color-coded boxes. This again illustrates the high resolution and flexibility of the method wherein any section of the image can be selected for detailed analysis and 3D rendering. Images of the toenail (red panel) are, to our knowledge, the first-ever images of a mouse toenail vasculature, a useful parameter because this is where ischemiainduced necrosis usually begins.

The cost of the procedure is $\$ 1-2$ per mouse and can be completed in an afternoon. The resolution around the FA is superior to micro-angiography and information on the vascular architecture approaches that of micro-CT.

\section{Acknowledgments}

Supported by NIH grants HL072924 (KAW) and F32DK08322 (MA) and by a Walter $\mathrm{G}$. Ross Chair in vascular biology (KAW). JB was supported by the Sheila and David Fuente Graduate Program in Cancer Biology, University of Miami. We acknowledge the University of Miami Sylvester Comprehensive Cancer Center Analytical Imaging Core Facility for all confocal imaging. This paper is subject to the NIH Public Access Policy.

\section{Competing interests}

The authors declare no competing interests

\section{References}

1. Abarbanell, A.M., J.L. Herrmann, B.R. Weil, Y. Wang, J. Tan, S.P. Moberly, J.W. Fiege, and D.R. Meldrum. 2010. Animal models of myocardial and vascular injury. J. Surg. Res. 162:239-249.

2. Powell, R.J. 2011. Update on biological therapies for critical limb ischemia. Cardiol. Clin. 29:411-417.

3. Emanueli, C., A. Caporali, N. Krankel, B. Cristofaro, S. Van Linthout, and P. Madeddu. 2007. Type-2 diabetic Lepr(db/db) mice show a defective microvascular phenotype under basal conditions and an impaired response to angiogenesis gene therapy in the setting of limb ischemia. Front. Biosci. 12:2003-2012.

4. Kang, J., H. Albadawi, V.I. Patel, T.A. Abbruzzese, J.H. Yoo, W.G. Austen, Jr., and M.T. Watkins. 2008. Apolipoprotein E-/- mice have delayed skeletal muscle healing after hindlimb ischemia-reperfusion. J. Vasc. Surg. 48:701-708.

5. Gounis, M.J., M.G. Spiga, R.M. Graham, A. 
Wilson, S. Haliko, B.B. Lieber, A.K. Wakhloo, and K.A. Webster. 2005. Angiogenesis is confined to the transient period of VEGF expression that follows adenoviral gene delivery to ischemic muscle. Gene Ther. 12:762-771.

6. Duvall, C.L., W.R. Taylor, D. Weiss, and R.E. Guldberg. 2004. Quantitative microcomputed tomography analysis of collateral vessel development after ischemic injury. Am. J. Physiol. Heart Circ. Physiol. 287:H302-H310.

7. Wagner, S., A. Helisch, T. Ziegelhoeffer, G. Bachmann, and W. Schaper. 2004. Magnetic resonance angiography of collateral vessels in a murine femoral artery ligation model. NMR Biomed. 17:21-27.

8. Hellingman, A.A., A.J. Bastiaansen, M.R. de Vries, L. Seghers, M.A. Lijkwan, C.W. Löwik, J.F. Hamming, and P.H. Quax. 2010. Variations in surgical procedures for hindlimb ischaemia mouse models result in differences in collateral formation. Eur. J. Vasc. Endovasc. Surg. 40:796-803.

9. Limbourg, A., T. Korff, L.C. Napp, W. Schaper, H. Drexler, and F.P. Limbourg. 2009. Evaluation of postnatal arteriogenesis and angiogenesis in a mouse model of hind-limb ischemia. Nat. Protocols 4:1737-1746.

10. Wang, R.K. and L. An. 2009. Doppler optical micro-angiography for volumetric imaging of vascular perfusion in vivo. Opt. Express 17:8926-8940.

11. Kumar, D., B.G. Branch, C.B. Pattillo, J. Hood, S. Thoma, S. Simpson, S. Illum, N. Arora, et al. 2008. Chronic sodium nitrite therapy augments ischemia-induced angiogenesis and arteriogenesis. Proc. Natl. Acad. Sci. USA 105:7540-7545.

12. Li, Y., Y. Song, L. Zhao, G. Gaidosh, A.M Laties, and R. Wen. 2008. Direct labeling and visualization of blood vessels with lipophilic carbocyanine dye DiI. Nat. Protocols 11:17031708.

13. Ertürk, A., C.P. Mauch, F. Hellal, F. Förstner, T. Keck, K. Becker, N. Jährling, H. Steffens, et al. 2011. Three-dimensional imaging of the unsectioned adult spinal cord to assess axon regeneration and glial responses after injury. Nat. Med. 18:166-171.

14. Abdulreda, M.H., G. Faleo, R.D. Molano, M. Lopez-Cabezas, J. Molina, Y. Tan, O.A. Echeverria, E. Zahr-Akrawi, R. RodriguezDiaz, P.K. Edlund, I. Leibiger, A.L. Bayer, V. Perez, C. Ricordi, A. Caicedo, A. Pileggi, P.O Berggren. 2011. High-resolution, noninvasive longitudinal live imaging of immune responses. Proc. Natl. Acad. Sci. USA 108:12863-12868.

15. Chalothorn D., J.A. Clayton, H. Zhang, D. Pomp, J.E. Faber. 2007. Collateral density, remodeling, and VEGF-A expression differ widely between mouse strains. Physiol. Genomics 30:179-191.

16. van Weel, V., M.M. Deckers, J.M. Grimbergen, K.L. van Leuven, J.H. Lardenoye, R.O. Schlingemann, G.P. van Nieuw Amerongen, J.H. van Bockel, et al. 2004. Vascular endothelial growth factor overexpression in ischemic skeletal muscle enhances myoglobin expression in vivo. Circ. Res. 95:58-66.

Received 2 May 2012; accepted 10 July 2012.

Address correspondence to Keith A. Webster, Department of Molecular and Cellular Pharmacology, Vascular Biology Institute,
University of Miami Miller School of Medicine, Miami, FL 33136. Email: kwebster@med.miami. edu

Supplementary material for this article is available at www.BioTechniques.com/article/113907

To purchase reprints of this article, contact: biotechniques@fosterprinting.com 